\title{
CORPOS FEMININOS MARCADOS PELA MASTECTOMIA
}

\author{
Ana Paula Leite de OLIVEIRA ${ }^{1}$ \\ Graça Rocha PESSOA ${ }^{2}$ \\ Andrezza Karinne Araújo de Medeiros PEREIRA ${ }^{2}$ \\ Ellany Gurgel Cosme do NASCIMENTO 3 \\ Amélia Carolina Lopes FERNANDES ${ }^{4}$ \\ Maria Irany KNACKFUSS ${ }^{4}$
}

'Enfermeira, Graduada no Campus Avançado "Prof ${ }^{a}$ Maria Elisa de Albuquerque Maia" - CAMEAM, Universidade do Estado do RioGrande do Norte - UERN, Pau dos Ferros/RN.

${ }^{2}$ Mestre, Docente do Curso de Graduação em Enfermagem no Campus Avançado "Profa Maria Elisa de Albuquerque Maia" - CAMEAM, Universidade do Estado do Rio Grande do Norte - UERN.

3Doutora, Docente do Curso de Graduação em Enfermagem no Campus Avançado "Prof" Maria Elisa de Albuquerque Maia" - CAMEAM, Universidade do Estado do Rio Grande do Norte - UERN.

${ }^{4}$ Doutora, Docente do Curso de Pós-Graduação "Mestrado em Saúde e Sociedade", Universidade do Estado do Rio Grande do Norte - UERN.

Autor para correspondência: Graça Rocha Pessoa. Endereço: Rua Antônio Januário, no 1.246. bairro: São Benedito. Cidade: Pau dos Ferros/RN. Telefone: (84)99652-1500. Email: gracarochauzl@ hotmail.com

Recebido em: 06/08/2015 - Aprovado em: 05/01/2016 - Disponibilizado em: 30/07/2016

\section{RESUMO}

Pesquisa de natureza qualitativa que teve como objetivo descrever as mudanças ocorridas na vida de mulheres acometidas por câncer de mama e mastectomizadas. As participantes da pesquisa constituíram-se de sete mulheres residentes na cidade de Pau dos Ferros/RN. Os dados foram coletados a partir da aplicação de um instrumento de entrevista semi-estruturada. Os depoimentos possibilitaram penetrar no mundo particular das mulheres que vivenciaram a experiência do câncer de mama, sobretudo a mastectomia, possibilitando identificar as transformações ocorridas nos aspectos físico, afetivo, relações familiares e sociais, na realização das atividades diárias e na auto-imagem destas mulheres. Para análise posterior dos dados, utilizamo-nos da análise de conteúdo, a partir da qual emergiram 04 categorias: O diagnóstico do câncer de mama confronta a mulher com o medo iminente da morte; A mastectomia afeta a concepção de beleza feminina e a auto-estima. A mastectomia traz mudanças na realização das atividades cotidianas; A mastectomia altera concepções e comportamentos da mulher. As mulheres vítimas de câncer de mama e submetidas a mastectomia sofrem importantes impactos sobre diversos aspectos das suas vidas, seja nos âmbitos social, afetivo, emocional e de relacionamento interpessoal.

Palavras-chave: Saúde feminina; Câncer de mama; Mastectomia.

\section{ABSTRACT}

Qualitative research that aimed to describe the changes in the lives of women affected by breast cancer and mastectomy. The study participants consisted of seven women residents in the city of Pau dos Ferros / RN. Data were collected from the application of a semi-structured interview tool. The testimonies made it possible to penetrate the private world of women who have experienced breast aner experience, especially mastectomy, allowing identify the changes occurred in the physical, emotional, family and social relationships, in carrying out daily activities and self-image of these women . For further analysis of the data, we use the content analysis, from which emerged 04 categories: The diagnosis of breast cancer confronts the woman with the imminent fear of death; Mastectomy affects the conception of female beauty and self-esteem; Mastectomy brings changes in the per for mance of daily activities; Mastectomy alters conceptions and behaviors of women. Women breast cancer victims an dunder going mastectomy suffe significant impacts onvarious aspects of their lives, whether in the social, affective, emotional and inter personal relationships.

Keywords: women's health; Breast cancer; Mastectomy. 


\section{INTRODUÇÃO}

O câncer de mama é o segundo tipo mais comum no mundo, representando $22 \%$ dos casos novos a cada ano. No Brasil tem se configurado como um problema de saúde pública, sendo a primeira causa de morte em mulheres no país. Segundo estimativas do INCA, em 2012 ocorrerão 52.680 casos novos.(BRASIL, 2012)

Os primeiros avanços no tratamento, surgem no primeiro século d.C, (DIAS, 1994) e hoje, apesar de todo o progresso técnicocientífico da medicina, e de milhares de métodos desenvolvidos para o diagnóstico e tratamento das doenças, ainda tem confrontado à saúde pública e as ciências biomédicas com suas manifestações agressivas e seu alto grau de morbidade e letalidade. Em decorrência disto continua sendo visto como uma "sentença de morte" pela maior parte das mulheres acometidas. De acordo com pesquisadores e estudiosos da área, é um acontecimento marcante com implicações físicas, sociais, psicológicas e sexuais. (CESNIK e SANTOS, 2012; NÁPOLES IZQUIERDO,et al, 2009; SCHWERZ FUNGHETTOLL, 2011;).

O aumento da ocorrência da doença nas diferentes populações no mundo, ainda não tem uma justificativa sólida, pois segundo estudiosos da área qualquer mulher está suscetível a ter câncer de mama em qualquer fase da sua vida, sendo mais comum entre mulheres de 40 a 60 anos de idade, e relativamente rara antes dos 35 anos. Quando descoberta precocemente apresenta maiores chances de sobrevida; a demora na obtenção do diagnóstico aumenta as chances para o poder invasivo das células cancerígenas. O risco de adoecimento de câncer de mama está relacionado à nuliparidade, menarca precoce, idade, primiparidade tardia, predisposição genética, obesidade, altura, menopausa tardia, fatores hormonais, história familiar, história de câncer, alimentação inadequada e consumo de álcool. E sua ocorrência ainda está associada, ao status sócio-econômico e cultural, fatores ambientais, processo de industrialização (agentes químicos, físicos, e biológicos), e outros fatores predisponentes como alimentares, hormonais, reprodutivos e constitucionais. (BRASIL, 2008).

São muitos os impactos do câncer de mama sobre a vida das mulheres, se apresentando sob vários aspectos, seja social, biológico, psicológico ou físico; nas fases pré, durante, e após o tratamento; é uma doença estigmatizante que nem sempre tem cura e necessita de tratamento longo e agressivo. Em inúmeros casos o tratamento é um meio de prolongar a vida da paciente e minimizar sofrimentos, uma vez que a resolução completa já não é mais possível.

Uma conseqüência de grande importância está relacionada ao próprio tratamento da doença - a mastectomia. As 
mulheres submetidas à mastectomia, podem passar por alterações de concepções, comportamentos e estilo de vida advindo de toda uma vivência, e pela construção de novas posturas, crenças, e estilo de vida favorável ou não para elas. (RODRIGUES; SILVA e MAMEDE, 2002).

Entendemos que não só a mulher, como também a mãe, o esposo, filhos e toda a família sofrem com o câncer de mama, acompanhando e convivendo com o adoecimento de um dos integrantes da família. Trata-se de um acontecimento de grande impacto sobre a estrutura familiar por não se reduzir apenas à dimensão física, mas atingir fortemente às dimensões psicológica, social e de percepção tanto da mulher acometida pela doença quanto pelos seus familiares. (TAVARES e TRAD, 2010).

A pesquisa teve como objetivo descrever o impacto da mastectomia na vida das mulheres submetidas a este tratamento.

\section{Material e Métodos}

Trata-se de pesquisa de caráter exploratório com abordagem qualitativa, desenvolvida com07 mulheres,residentes na cidade de Pau dos Ferros/RN, vítimas de câncer de mama e submetidas a mastectomia, entre o período de 2005 a 2010.A pesquisa foi realizada entre janeiro a dezembro de 2011. A faixa etária das participantes varia entre 42 a
77 anos, com predominância de mulheres com idade acima de 50 anos.

Os critérios de inclusão foram: que o tempo transcorrido entre a mastectomia e a aplicação da entrevista não fosse superior a 10 anos; que a mulher residisse em Pau dos Ferro/RN; estivesse adscrita na Estratégia Saúde da Família da Unidade São Judas Tadeu e que aceitasse de livre arbítrio participar da pesquisa assinando o Termo de Consentimento Livre e Esclarecido (TCLE).Como critérios de exclusão tivemos: mulheres que apresentassem deficiência mental e que não atendessem aos critérios de inclusão. As participantes selecionadas encontravam-se adscritas na Unidade de Saúde da Família São Judas Tadeu, localizado no município de Pau dos Ferros/RN. A escolha desta unidade deu-se a partir do conhecimento empírico sobre a predominância do número de mulheres mastectomizadas nesta microárea.

Como instrumento de coleta de dados, utilizamos uma entrevista semi-estruturada e para análise dos dados a técnica de análise de conteúdo, a qual, constitui-se em "Um conjunto de técnicas de análise de comunicação visando obter, por procedimentos sistemáticos e objetivos de descrição do conteúdo das mensagens, indicadores (quantitativos ou não) que permitam a inferência de conhecimentos relativos às condições de produção/recepção destas mensagens" (MINAYO, 2004). 
A pesquisa foi aprovada pelo Comitê de Ética e Pesquisa (CEP) da instituição proponente, sob o Protocolo 121/10, $\mathrm{n}^{\circ}$ CAAE 0108.0.428.000-10, atendendo a Resolução CNS 196 do Ministério da Saúde.

\section{RESULTADOS}

O conteúdo desta pesquisa foi baseado na experiência de sete mulheres vítimas de câncer de mama e da mastectomia. A maioria é natural de Pau dos Ferros, sendo uma do Maranhão, uma do Francisco do Oeste, e a outra de Recife/PE. Todas as participantes moram na área de abrangência da Estratégia Saúde da família São Judas Tadeu, localizado na cidade de Pau dos Ferros, e foram submetidas a cirurgia de mastectomia total ou parcial entre o ano de 2005 e 2010.

A faixa etária das participantes varia entre 42 a 77 anos, com predominância de mulheres com idade acima de 50 anos; quanto a cor, todas se consideram de cor branca. Quanto a religião quatro disseram ser católicas, mas não praticantes, duas católicas praticantes e somente uma referiu ser cristã, não especificando sua religião. Quanto ao grau de instrução todas são alfabetizadas, sendo duas com $3^{\circ}$ grau completo, duas com $2^{\circ}$ grau completo, uma com o ensino fundamental incompleto, uma com primário completo e uma com o primário incompleto.
Em relação ao estado civil, três delas são casadas, duas divorciadas, uma solteira e uma viúva. Apresentando uma composição familiar muito heterogênea, sendo formados por filhos, marido, irmãos, netos, e funcionários. $60 \%$ têm de um a quatro filhos, $30 \%$ tem seis a sete filhos, e $10 \%$ não tem filhos.

Do total de mulheres, uma é do lar, duas são agricultoras, uma auxiliar de enfermagem, uma ambientalista, uma vendedora ambulante (autônoma) e uma artesã. A maioria já é aposentada $(57,1 \%)$, as demais continuam exercendo suas atividades de acordo com suas condições físicas, pois segundo a maior parte das participantes a mastectomia comprometeu a sua condição físicadificultando a realização de alguns trabalhos, inclusive o doméstico.

As participantes pertencem à classe média e baixa. $70 \%$ das mulheres tem renda individual de zero a um salário mínimo; $30 \%$ tem dois salários mínimos. $100 \%$ temrenda familiar de dois a quatro salários mínimos.

Quanto ao tratamento,as mulheres foram distribuídas entre as modalidades de radioterapia, quimioterapia e hormonioterapia, obedecendo a um dos princípios de tratamento do câncer, na atualidade que é a combinação de intervenções diferenciadas a fim de se obter melhores taxas de cura e/ou sobrevivência. 
Porém duas mulheres não concluíram o física, fisiológica e emocional. (Tabela 1) tratamento, justificando falta de condição

Tabela 1 - Distribuição de mulheres conforme tratamento adjuvante a mastectomia.

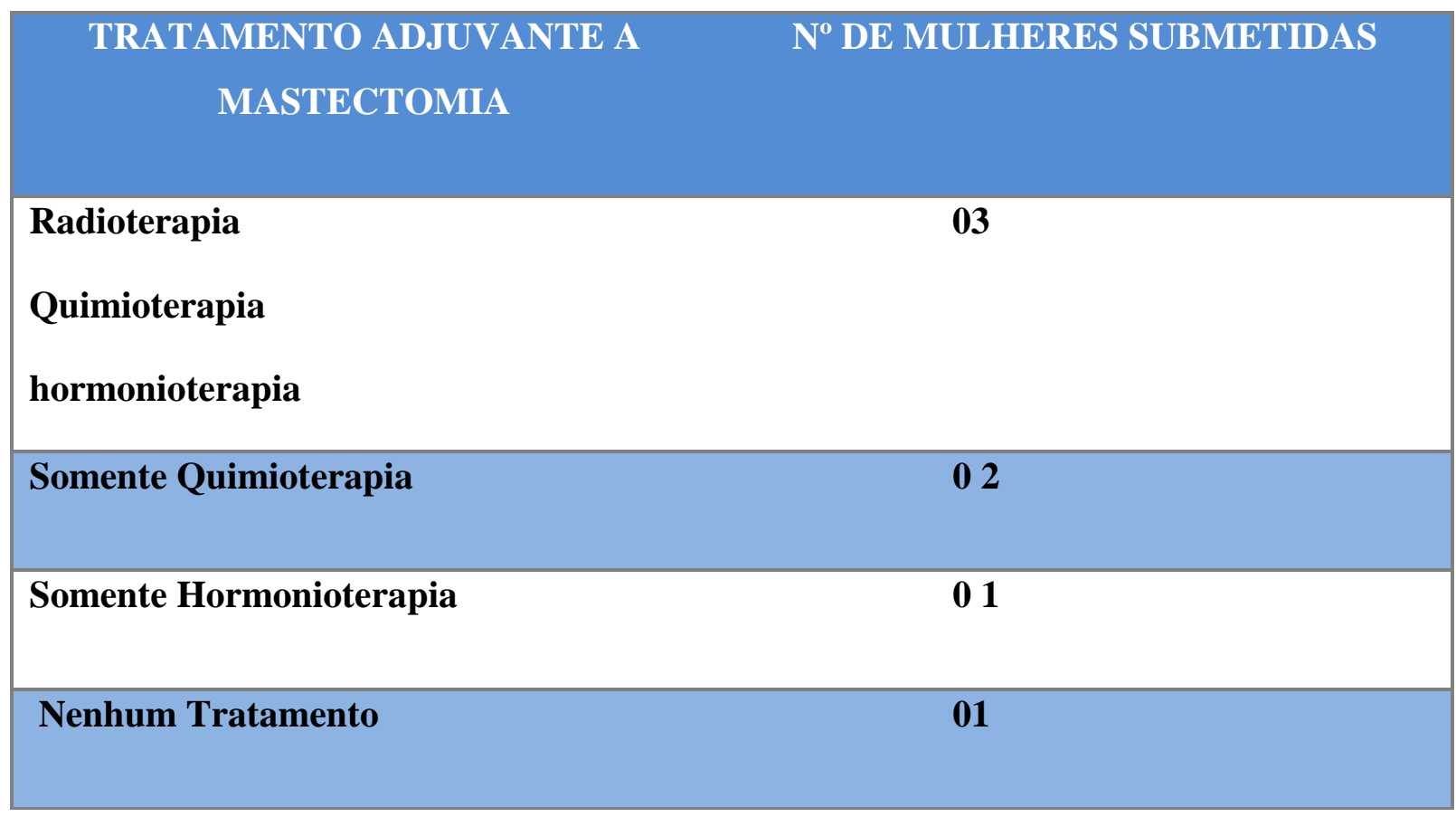

A partir do levantamento de dados chegamos a construção das seguintes categorias analíticas que explicam o impacto do câncer de mama e da mastectomia sobre a vida das mulheres acometidas:

\section{O diagnóstico do câncer de mama} confronta a mulher com o medo iminente da mortee a angústia de tornar-se mastectomizada

Entre as participantes da pesquisa três $(42,8 \%)$ relataram que ao serem comunicadas sobre o diagnóstico e a cirurgia de mastectomia, reagiram com muita tranqüilidade e confiança, aceitando a mastectomia com normalidade.

"Minha reação foi normal. Não tive desespero nenhum. Até o médico ficou com medo de me dar a noticia, por causa da minha irmã que morreu com câncer de mama, mas ela foi que não cuidou. Mas a minha reação foi normal." (cravo) 
Após receberem o diagnóstico do câncer de mama, as mulheres estavam dispostas a se submeterem a qualquer tratamento, mesmo esse sendo a mastectomia, castração de um órgão tão cheio de representações para a sexualidade feminina.

As demais participantes $(57,1 \%)$ revelaram sentimentos de angústia, ansiedade, medo do desconhecido e, especialmente medo da morte. $\mathrm{O}$ diagnóstico do câncer de mama foi recebido como uma sentença de morte.

Para as mulheres o diagnóstico do câncer de mama é mais aterrorizante, do que a perda da mama; levando-as a sentirem a necessidade de retirar o tumor o mais rápido possível. Embora a mastectomia represente um momento de significativa ansiedade para amulher, ao mesmo tempo simboliza a possibilidade de cura. (ALVES, P. C.et al, 2010 e SILVA; JENERAL e DUARTE, 2008).

Neste momento não importam muito as consequências do tratamento. Porém, o alívio transitório, produzido pela possibilidade de resolução da doença, tem fim num curto período de tempo quando a mulher se conscientiza da sua realidade. Superado o medo da morte, vivenciado no primeiro estágio de enfrentamento da doença, a mulher retorna ao convívio social e as atividades laborais vindo à tona as preocupações com o corpo, trazendo uma série de modificações na sua auto-imagem e nos relacionamentos afetivos de modo geral. (AZEVEDO e LOPESI, 2010).

\section{A mastectomia afeta a concepção de beleza feminina e a auto-estima.}

Amutilação damama é uma experiência única e inesquecivel. Os relatos das entrevistadas demonstraram claramente que a doença provocou uma série de mudanças em suas vidas, modificando sobremaneira a sua auto-imagem e a percepção da vida.

As mulheres revelam insatisfação quanto à percepção corporal e não aceitação da perda da mama. Classificam sua imagem como alterada e estranha, e demonstram sentimentos de inferioridade e impotência: Muito esquisito não gosto de olhar no espelho, sinto diferente. Meu corpo mudou muito. (Rosa Amélia)

$\mathrm{O}$ primeiro contato que elas estabelecemcom o seu corpo operado diante do espelho, com a ausência de uma das mamas, provoca um sentimento de estranheza e sofrimento. A imagem corporal é sobremaneira prejudicada pela mastectomia. (MAJEWSKI et al, 2012).

As entrevistadas referiram sentirem-se incomodadas com a falta da mama, relatam que o corpo feminino com uma única mama fica descompensado e assimétrico, gerando sentimentos de inferioridade em relação às 
outras mulheres, apresentando baixa autoestima e dificuldades para aceitar sua nova condição física. A sexualidade feminina, fenômeno abrangente envolvendo o sexo, o prazer, o desejo, a auto-imagem, a aceitação do corpo, a sensação de bem estar consigo mesma e com a segurança é comprometido na vida das mulheres mastectomizadas. (CESNIK e SANTOS, 2012).

\section{A mastectomia traz mudanças na realização das atividades cotidianas.}

Entre as participantes, quatro (57\%) relataram quehouverammudanças na realização das atividades diárias; entre elas o impedimento para desempenhar atividades rotineiras, como: lavar roupa e passar, lavar louça e cuidar da casa:

A mastectomia trouxe mudanças em tudo. Não posso fazer mais nada de trabalho doméstico. Tenho que varrer pouquinho, porque o braço fica inchado, não posso cozinhar e engomar porque tenho medo de me queimar, não posso costurar com medo de me furar, não posso pegar peso. Mudei meu estilo de roupa e muitas outras coisas. ( Lirios)

As entrevistadas revelam insatisfação e mal-estar com as alterações físicas, emocionais, e principalmente com as limitações e proibições na realização das atividades diárias pós-mastectomia. Relatam mudanças na sua condição física $\mathrm{e}$ comportamental.Revelam o mal- estar provocado pela sensação de impotência e déficit de autonomia. Assim, a mastectomia provoca um conjunto de alterações quer nas rotinas, comportamentos, atitudes, responsabilidades e competências da mulher e família.

\section{A mastectomia altera concepções e comportamentos da mulher.}

A ocorrência do câncer de mama e consequente mastectomia induz a consciência da necessidade de modificar determinados hábitos, o processo de adoecer leva a mulher a uma valorização da vida e do cuidado consigo mesma, priorizando o seu desenvolvimento pessoal. (GURGEL, 2009).

Ao se deparar com uma doença grave, elas são induzidas a buscar o autoconhecimento, a avaliar sua postura ante as situações da vida, a resgatar alguns valores 
e princípios muitas vezes esquecidos em função dos interesses materialistas que norteavam suas vidas até aquele momento.

A experiência com o câncer de mama e o tratamento que eu tive que fazer melhorou a minha forma de ver a vida. Tô vivendo agora, mais intensamente.

(Orquídea)

Desse modo, ao determinar novas perspectivas de vida à mulher ultrapassa os limites da doença. Reconstitui sua identidade dentro de um novo conceito de si mesma, formando e reformulando concepções e comportamentos, aceitando-se como uma mulher portadora de câncer de mama. E na tentativa de entender o motivo do câncer, e para se adaptar à situação e conviver com a doença, a mulher descobre-se forte, e utilizase de estratégias como a fé:

Deus me deu oportunidade de lutar, me deu mais uma chance de vida. Me deu a cura, me deu mais uma chance. Todas as mulheres que passam por isso devem lutar contra a doença e nunca baixar a cabeça diante do problema, encarar com naturalidade, nunca com desespero. O desespero não leva a nada. (Cravo)

Os depoimentos das mulheres evidenciam o sofrimento, as dificuldades enfrentadas por elas na experiência com o câncer de mama. Tendo que adaptar-se a uma nova situação e buscar uma nova percepção de si mesma com novas perspectivas. O enfrentamento do problema possibilita reverter à situação estressante em esperança, encontrar alternativas que lhe proporcionem conforto emocional e que ajudem no aprendizado de novas habilidades e comportamentos.

Segundo Vieira, Gomes e Trajano, (2005, p.106) O tratamento de câncer segue uma sequência que vai desde o sofrimento, passando pela superação dos problemas, até chegar a uma crença na possibilidade de cura. Desta forma, a imagem cruel do câncer pode ser atenuada e, em algumas vezes, fazendo com que a vida seja reinventada.

\section{DISCUSSÃO}

A realidade do diagnóstico e a necessidade da mastectomia confrontam a 
mulher com diversos conflitos de fórum íntimo, feminino e emocional. Neste primeiro momento, o fator de grande impacto sobre a vida da mulher é a compreensão do diagnóstico como uma sentença de morte. (MOURA, 2010).

Esta experiência com o sentimento do medo da morte, obscurece, por algum tempo, a visão feminina sobre a própria mastectomia; por um instante, esta parece insignificante perante a gravidade da doença.Embora a mastectomia represente um momento de significativa ansiedade para a mulher, ao mesmo tempo simboliza a possibilidade de cura. (ALVES, P. C.et al, 2010 e e SILVA; JENERAL e DUARTE, 2008).

\section{Neste primeiro estágio de} enfrentamento da doença não importam muito as consequências do tratamento. Porém, o alívio transitório, produzido pela possibilidade de resolução da doença, tem fim num curto período de tempo quando a mulher se conscientiza da sua realidade. Superado, em parte, o medo da morte, a mulher retorna ao convívio social e as atividades laborais vindo à tona as preocupações com o corpo, a auto-imagem, as relações sociais e afetivas e o retorno às atividades laborais. (AZEVEDO e LOPESI, 2010).

Nesta sequência, ela passa a experimentar com mais intensidade o impacto do câncer e da mastectomia sobre a sua vida nos diversos aspectos, seja na auto-magem, no aspecto afetivo-sexual, na realização das atividades da vida diária ou laborais e na sua própria concepção sobre a vida.

A mulher aprendeu durante toda a sua vida que as mamas além de desempenharem a função fisiológica, que se reflete na amamentação, também representam culturalmente um símbolo de identidade expressa pela sensualidade e sexualidade. (SILVA, 2010). Ao ser submetida amastectomia, sente dificuldade em aceitar sua nova condição física. A amputação de parte ou de toda a mama representa uma situação traumática, potencializada pelos significados psicológicos e culturais atribuídos aos órgãos femininos. (OIZ, 2005).

Em estudo realizado por OLIVEIRA et. al. (2006), aplicando a Escala de Autoestima de Rosemberg, os autores chegaram ao resultado que as mulheres mastectomizadas, seja parcial ou totalmente, sofrem um impacto sobremaneira negativo sobre a sua autoestima. (OLIVEIRA, 2006).

Consequentemente a vida afetivosexual destas mulheres também sofre modificações negativas em decorrência da mutilação sofrida e sentimento de dependência e perda da feminilidade. (SCHWERZ FUNGHETTOLL, 2011). Algumas mulheres podem sofrer um comprometimento tão importante da autoestima a ponto de se sentirem repulsivas e evitam contatos sexuais. (CESNIK e SANTOS, 2012). 
A vida social da mulher é modificada, no momento em que esta tem que adaptar todo o seu estilo de vida de acordo com as restrições do tratamento; cumprir dietas alimentares, evitar o consumo de álcool e uso de certas medicações, uso de roupas apertadas ou escuras, evitar noites acordadas, esforços, abandonar o emprego ou se afastar por tempo indeterminado; restringir suas atividades domésticas e sociais para realizar um tratamento, algumas vezes, com poucas perspectivas de cura. (ALMEIDA, 2001 e GONÇALVES, 2012).

Em estudo comparativo entre cirurgia radical e conservadora os autores encontraram no grupo submetido a cirurgia radical um maior prejuízo quanto ao desenvolvimento das atividades laborais. Apenas $40 \%$ deste grupo conseguiu retornar às suas atividades laborais, enquanto $63,3 \%$ das mulheres submetidas a cirurgia conservadora conseguiram retomar suas atividades.

Para reconhecer as mudanças ocorridas na vida é necessário que a mulher se desprenda da racionalidade e reconheça que a doença trouxe alterações sejam elas físicas, emocionais ou sociais. E para isso a mulher tem que resgatar sua vida antes do adoecimento e refletir sobre as condutas e a sua personalidade. E do mesmo modo os serviços e profissionais de saúde devem estar preparados para a oferta de uma assistência mais integradora a estas mulheres que lhes deem suporte para o enfrentamento dos desafios que o diagnóstico de câncer de mama e seu tratamento vão impor-lhes.

\section{CONSIDERAÇÕES FINAIS}

Através desta pesquisa foi possível confirmar o impacto que o câncer de mama e a mastectomia produzem na vida da mulher e de sua família. Este acontecimento impacta a vida das mulheres em todos os aspectos: físicos, social, emocional e afetivo-sexual.

Conforme tem demostrado diversas pesquisas que foram referenciadas, aqui, este trabalho aponta, de modo semelhante, que a mastectomia radical constitui-se num tratamento deveras agressivo para o ser feminino, alterando negativamente toda a dinâmica da vida da mulher e de sua família.

Tais alterações podem ser percebidas nas mudanças negativas quanto a autoimagem da mulher, no sofrimento psíquicoemocional provocado tanto pelo diagnóstico da doença quanto pela perda da mama;na deterioração das relações afetivas e nas mudanças negativas quanto à vivência da sexualidade e nas dificuldades para o restabelecimento das atividades laborais, comprometendo, de modo geral a qualidade de vida destas mulheres.

Mediante a intensidade que a mastectomia compromete a saúde e vida das mulheres espera-se que a pesquisa contribua na elaboração de programas e políticas de 
saúde que qualifiquem a assistência a saúde da mulher com câncer de mama nos aspectos fisiológico, psicológico, e social. E que os profissionais de saúde, sejam instrumentalizados a fim de estarem preparados para o atendimento às reais necessidades de saúde deste grupo, amenizando o sofrimento e promovendo o bem estar da melhor forma possível.

Destacamos também que as mulheres submetidas amastectomia necessitam não somente da assistência de saúde oferecida por profissionais, mas também do apoio incontestável da família, devendo essa ser solidária, não permitindo que a paciente se deixe vencer pela doença, acompanhá-la sempre em todos os momentos, estimulá-la a realizar o tratamento e a lutar pela vida.

\section{REFERÊNCIAS}

ALMEIDA, A. M. deet al. Construindo o significado da recorrência da doença: a experiência de mulheres com câncer de mama. Revista Latino-Americana de Enfermagem, v. 9, n. 5, p. 63-69, 2001.

ALVES, P. C.et al. Conhecimento e expectativas de mulheres no pré-operatório da mastectomia. Revista da Escola de Enfermagem da USP, v. 44, n. 4, p. 989-995, 2010.

AZEVEDO, R. F.; LOPESI, R. L. M. Concepção de corpo em Merleau-Ponty e mulheres mastectomizadas. Revista

Brasileira de Enfermagem, v. 63, n. 6, p. 1067-1070, 2010.
BRASIL. Ministério da Saúde. Instituto Nacional do Câncer. Estimativa 2012: Incidência de câncer no Brasil. (internet). Rio de Janeiro: INCA. Disponível em: </www.inca.gov.br >. Acesso em: 04 set 2012.

BRASIL, Ministério da Saúde. Instituto Nacional do Câncer. Ações de enfermagem para o controle do câncer: uma proposta de integração ensino-serviço. Rio de Janeiro (RJ): Ministério da Saúde/Instituto Nacional do Câncer, 2008.

CESNIK, V. M.; SANTOS, M. A. D. Mastectomia e sexualidade: uma revisão integrativa. Psicologia: Reflexão e Crítica, Porto Alegre, v. 25, n. 2, p. 339-349, 2012.

DIAS, E. N. A mastologia através da história. In: DIAS, E.N. et. al. Mastologia Atual. Rio de Janeiro: Editora Revinter, 1994. p. 3-6.

GONÇALVES, S.R. de O. S.; ARRAIS, F. M. de A.; FERNANDES, A. F. C. As implicações da mastectomia no cotidiano de um grupo de mulheres. Revista da Rede de Enfermagem do Nordeste-Rev Rene, v. 8, n. 2, 2012.

GURGEL, M. S. C. Qualidade de vida e sexualidade de mulheres tratadas de câncer de mama. RevBrasGinecolObstet, v. 31, n. 2, p. 61-7, 2009.

MAJEWSKI, J. M.et al. Qualidade de vida em mulheres submetidas à mastectomia comparada com aquelas que se submeteram à cirurgia conservadora: uma revisão de literatura. Ciência \& Saúde Coletiva, v. 17, n. 3, p. 707-716, 2012.

MINAYO, MCS. O desafio do conhecimento: pesquisa qualitativa em saúde. 2004. 8.ed. São Paulo: Hucitec, 269p. 
MOURA, F. M. J. S. P.et al. Os sentimentos das mulheres pós-mastectomizadas. Esc Anna Nery, v. 14, n. 3, p. 477-84, 2010.

NÁPOLES IZQUIERDO, Y. et al. Cirugía conservadora y radical enestadíostempranosdelcáncer de mama.

Revista Archivo Médico de Camagüey, v. 13, n. 3, p. 0-0, 2009.

OIZ, B. Reconstrucción mamaria y beneficio psicológico. Analesdel Sistema Sanitario de Navarra, Pamplona, v. 28, supl. 2, p. 19-26, 2005.

OLIVEIRA, A. C. et al. Mastectomia: impacto auto-estima das pacientes. Rev. bras. mastologia, v. 16, n. 4, p. 156-160, 2006.

RODRIGUES, D. P.; SILVA, R. M. da; MAMEDE, M. V. Analisando o processo adaptativo no autoconceito da mulher mastectomizada. Nursing, São Paulo, v. 5, n. 51, p. 29-34, 2002.

SILVA, M. C.; JENERAL, R. B. R.;

DUARTE, L. R. Mulheres mastectomizadas: estranhas no ninho. Nursing, São Paulo, v. 11, n. 122, p. 333-338, 2008.

SILVA, S. E.D. da et al. Representações sociais de mulheres mastectomizadas e suas implicações para o autocuidado. Revista Brasileira de Enfermagem, v. 63, n. 5, p. 727-34, 2010.

SCHWERZ FUNGHETTOLL, S. Nossa vida após o câncer de mama: percepções e repercussões sob o olhar do casal. Revista Brasileira de Enfermagem, v. 64, n. 3, p. 536-44, 2011. Disponível em: http://www.scielo.br/pdf/reben/v64n3/v64n3a 18.pdf. Acesso em: 04 set 2012.

TAVARES, J. S. C.; TRAD, L. A. B. Estratégias de enfrentamento do câncer de mama: um estudo de caso com famílias de mulheres mastectomizadas. Ciênc. saúde coletiva, Rio de Janeiro, v. 15, n. 1, 2010. 